\title{
Definitive Screening Designs and latent variable modelling for the optimization of solid phase microextraction (SPME): Case study - Quantification of volatile fatty acids in wines
}

\author{
Ana C. Pereira ${ }^{a, b}$, Marco S. Reis ${ }^{a, *}$, João M. Leça ${ }^{\text {b,c }}$, Pedro M. Rodrigues ${ }^{b}$, José C. Marques ${ }^{\text {b,c }}$ \\ ${ }^{\text {a } C I E P Q P F}$, Department of Chemical Engineering, University of Coimbra, Pólo II - Rua Sílvio Lima, 3030-790, Coimbra, Portugal \\ ${ }^{\mathrm{b}}$ Faculty of Exact Sciences and Engineering, University of Madeira, Campus da Penteada, 9000-390, Funchal, Portugal \\ ${ }^{\mathrm{c}}$ Institute of Nanostructures, Nanomodelling and Nanofabrication (I3N), University of Aveiro, Aveiro, Portugal
}

\section{A R T I C L E I N F O}

\section{Keywords:}

Definitive Screening Designs

Principal component analysis

HS-SPME

Optimization

Volatile fatty acids

Fortified wines

Weighted least squares

\begin{abstract}
A B S T R A C T
In the present study, we apply the recently proposed Definitive Screening Designs (DSD) to optimize HS-SPME extraction in order to analyze volatile fatty acids (VFA) present in wine samples. This is the first attempt to apply this new class of designs to one of the most well-known and widely applied extraction techniques. The latent structure of the responses is also explored for defining the optimal extraction conditions. DSD is a new screening design with the potential to significantly reduce the number of experiments required to estimate the model parameters and to establish the optimum operation conditions. Therefore, there is an obvious interest in assessing the benefits of DSD in practice. In this work, this design framework is applied to the simultaneous optimization of seven extraction parameters (responses). Both qualitative and quantitative extraction parameters are considered, in order to test the flexibility of DSD designs: a two-level qualitative variable, the fiber coating, and six quantitative variables, namely the pre-incubation time, the extraction time and temperature, the headspace/sample volume, the effect of agitation during extraction and the influence of the ethanol content (sample dilution). Optimization of analytes' chromatographic responses was carried out both individually (response by response) and altogether, by modelling the responses in the latent variable space (i.e., explicitly considering their underlying correlation structure). In the end, a consensus analysis of all perspectives was considered in the definition of the overall optimal extraction conditions for the quantification of VFA in fortified wines. The solution found was to use a DVB/Car/PDMS fiber, $10 \mathrm{~mL}$ of samples in $20 \mathrm{~mL}$ vial, $40 \mathrm{~min}$ of extraction at $40{ }^{\circ} \mathrm{C}$. The analysis also revealed that the factors incubation time, agitation and sample dilution do not play a significant role in explaining the variability of extraction parameters. Therefore, they were set to the most convenient levels. The methodology followed was thoroughly validated and the following figures of merit were obtained: good linearity $\left(\mathrm{R}^{2}>0.999\right.$, for all compounds), high sensitivity (LOD and LOQ are close or below the values found in literature), recoveries of approximately $100 \%$ and suitable precision (repeatability and reproducibility lower than $7.21 \%$ and $8.61 \%$, respectively). Finally, the optimized methodology was tested in practice. Several wine samples were analyzed and the odor activity value calculated to facilitate the identification of their importance as odor active compounds in different aged fortified wines. This work demonstrates the benefits of using DSD and latent variable modelling for the optimization of analytical techniques, contributing to the implementation of rigorous, systematic and more efficient optimization protocols.
\end{abstract}

\section{Introduction}

Definitive Screening Designs have been attracting a considerable interest in several communities strongly involved in the active collection and analysis of experimental data. This new class of screening designs requires only one more experiment than twice the number of factors under analysis, and still allows to estimate the main effects without any aliasing with each other or with two-factor interactions (in fact, they were incidentally discovered while searching for designs with good aliasing properties through optimal DOE computational approaches). An

\footnotetext{
* Corresponding author.

E-mail address: marco@eq.uc.pt (M.S. Reis).
} 


\begin{tabular}{|c|c|}
\hline \multicolumn{2}{|c|}{ Abbreviations } \\
\hline DOE & Design of experiments \\
\hline DSD & Definitive Screening Designs \\
\hline HS-SPME & Headspace solid-phase microextraction \\
\hline KI & Kovats indexes \\
\hline LOD & Limit of detection \\
\hline LOQ & Limit of quantification \\
\hline OAV & Odor activity value \\
\hline OFAT & One-factor-at-a-time \\
\hline PCA & Principal Component Analysis \\
\hline VFA & Volatile fatty acids \\
\hline WLS & Weighted least squares regression \\
\hline
\end{tabular}

interesting feature of DSDs is that, contrary to classical screening designs, pure quadratic effects can also be estimated, if the number of factors is greater than 6 and their strength is large enough to be detected [1]. This is granted by the existence of center points in every row of the design and by the particular combination of levels used in the design matrix. This makes DSD a three-level screening design, but whose levels are not set following the classical Response Surface Methods (the design may not be orthogonal in general, but for some number of factors it is indeed orthogonal for the main effects). The aforementioned low number of runs required, roughly twice as many as the number of quantitative factors under optimization [2-4], is of course of significant interest to practitioners. When compared with classical fractional factorial designs, DSD's have the advantage of estimating independently the main and the quadratic effects (without confounding), as well as of being unaliased with two-factors interactions. However, the literature still lacks information on the application of DSD to real world applications and the sensitivity of these designs to capture the relevant effects. Therefore, in this work we set the goal to assess the application of DSD to HS-SPME optimization, given the relevancy of this technique.

The present case study regards the quantification of Volatile Fatty Acids in wines through the application of HS-SPME. Seven extraction factors are considered in the optimization of the HS-SPME procedure for quantification of nine volatile fatty acids (VFA) in wines (whose chromatographic responses constitute the outputs of the analysis), namely: isobutyric acid, butyric acid, isovaleric acid, valeric acid, hexanoic acid, octanoic acid, nonanoic acid, decanoic acid and dodecanoic acid. So far, only Ref. [5] reports a procedure where HS-SPME coupled to gas chromatography with a mass spectrometry detector (GC-MS) is used for the quantification of volatile fatty acid in wines. The present study, considers the analysis of two additional parameters in the extraction procedure, namely the pre-incubation time and ratio of headspace/sample volume, besides a completely different optimization and data analysis procedure. The remaining variables analyzed were fiber, time and extraction temperature, the effect of agitation during extraction and the influence of the ethanol content (sample dilution). Given the existence of multiple responses (the chromatographic profiles of the nine VFAs), which are furthermore strongly correlated, our analysis included a latent variable modelling approach for describing the dominating correlation structure of the responses and to analyze the simultaneous impact of the design factors over the chromatographic responses of the analytes. The analysis conducted led to an optimal consensus solution, which was then validated and finally applied to new samples, on order to demonstrate its effectiveness.

The reminding parts of this article are organized as follows. Section 2 revises the application of Headspace Solid-Phase Microextraction (HSSPME) for the analysis wine. Section 3 presents the relevant experimental information of the study, including a description of the samples, methods and instrumentation used. The fourth section is dedicated to a brief description of DSD and the latent variable modelling framework used for data analysis. The results obtained are presented and discussed in Section 5. The validation of the HS-SPME method and its application to new samples are reported in Section 6. The article is closed with a summary of the main conclusions about the methodological aspects (DSD and latent variable modelling for optimizing the extraction conditions) and the results obtained in the case study (Section 7).

\section{Headspace Solid-Phase Microextraction (HS-SPME) for the analysis of volatile compounds in wine}

Headspace solid-phase microextraction (HS-SPME) is a state of the art extraction methodology in the analysis of volatiles compounds in wines. Its application in this context was recently reviewed in Ref. [6], where it was highlighted the good sensitivity and accuracy of HS-SPME methodologies, together with the advantages of being solvent-free and requiring only limited manipulation of the samples. The additional possibility of full automation makes it one of the first choices in this experimental domain. In the same review paper the authors underline the critical importance of conducting an appropriate optimization of the HS-SPME process, an aspect that is often overlooked and remains to be properly addressed: most of the times the parameters are optimized through simple one-factor-at-a-time (OFAT) approaches, where one factor is optimized in turn, and fixed from then on. This OFAT procedure prevents the identification of any relevant interactions among factors, a limitation that cannot be underestimated in real world applications. Therefore, the final solutions found with OFAT procedures end up being sub-optimal, at best. The disadvantages of OFAT optimization are well-known and documented in the Design of Experiments (DOE) literature, and have been thoroughly discussed elsewhere [7-15], being now widely recognized its limitations and the fact that it can lead to suboptimal results concerning the efficiency of the methodology [16].

The reluctance to adopt and applying DOE methodologies in wine volatile analysis contrasts with other application fields of HS-SPME in analytical chemistry, in which this practice is already part of method development workflow $[9,10,17-23]$. In these cases, the number of parameters involved in the optimization phase is in general in the range of $3-5$ [24-46], being the time and temperature of extraction the parameters most frequently considered, followed by the salting out effect, the ratio of sample/headspace volume and the stirring influence. Although fewer in number, there are also other works that include in the optimization phase other HS-SPME parameters with known influence in the extraction process, namely the fiber coating [47], the pre-incubation time and temperature [47-49], the desorption time and temperature [49-51] and the derivatization conditions in situations when this process is also involved [49,52].

The choice of the particular experimental design methodology to be used depends on the objectives of the study and the nature of variables. Generally, the DOE methodologies are classified as screening, process characterization, matching target, optimization and robustness designs, being screening and optimization the prevailing classes in practical applications. Screening is focused in the identification of the main factors involved in the phenomena, separating the "critical few" from the "trivial many". Optimization, on the other hand, addresses the definition of the factor levels that optimize a given criterion. In the scope of HS-SPME tuning, the class of full factorial $[29,36]$ and fractional factorial designs (with resolution IV and V) [25, $33-35,39,42,44]$ are the most often used approaches. As to optimization, the response surface methodologies (RSM), namely the Central Composite design [26,30-32,37,38,52] and the Doehlert designs [27], together with the algorithmic Optimal designs [28,45,47,48,53], are the dominant techniques for establishing the optimal settings for each factor. Other studies also adopt both categories of designs in a sequential manner, first identifying the important factors and then looking for their optimum settings $[24,40,41,43,46,49,50]$. 


\section{Experimental details}

This section presents information regarding the chemical materials and wine samples used in this study, followed by a subsection describing the HS-SPME parameters selected to be optimized and their corresponding operating ranges. Finally, the chromatographic conditions adopted are presented as well as details on the final validation of the proposed analytical methodology.

\subsection{Chemicals and materials}

The volatile fatty acids isobutyric, butyric, hexanoic and decanoic acid were supplied from Aldrich chemistry (Steinheim, Germany), the valeric acid, isovaleric acid, nonanoic acid and dodecanoic acid were obtained from Acros Organics (New Jersey, USA) and the octanoic acid was obtained from SAFC (St. Louis, MO, USA). All these standards have a purity grade of more than $97.0 \%$, adequate to GC-MS analyses. The absolute ethanol, with purity higher than $99.8 \%$, was purchased from Sigma-Aldrich (Steinheim, Germany). The 3-octanol standard was supplied from Acros Organics with a purity of $99.5 \%$ (Loughborough, UK). Sodium chloride and sodium hydroxide were supplied by Panreac Química S.A. (Barcelona, Spain), with the purity of $99.5 \%$ and $98.0 \%$, respectively, and the tartaric acid from Merck (Darmstadt, Germany) with the purity of $99.5 \%$. The alkane solution (C7 - C30) was obtained from Supelco (Sigma Aldrich, St. Louis, MO, USA).

Synthetic wine was prepared for the validation of the analytical methodology using a solution of $6 \mathrm{~g} / \mathrm{L}$ of tartaric acid, a content of $18 \%$ ethanol and the $\mathrm{pH}$ adjusted to 3.50 with $\mathrm{NaOH} 1 \mathrm{M}$. A stock solution of about $10 \mathrm{~g} / \mathrm{L}$ was prepared for each VFA in ethanol. Then, suitable dilutions of the stock solutions were prepared in synthetic wine, to obtain the intermediate solutions of $100 \mathrm{mg} / \mathrm{L}$. Afterwards, working solutions $(\mathrm{n}=7)$ were prepared by dilution from standard solutions, within the validation range $0.01 \mathrm{mg} / \mathrm{L}$ up to a maximum of $8.0 \mathrm{mg} / \mathrm{L}$.

The SPME fibers tested were purchased from Supelco (Bellefonte, PA, USA), namely the stableflex fiber core coated with $50 / 30 \mu \mathrm{m}$ divinylbenzene-carboxen-polydimethylsiloxane (DVB/Car/PDMS) and the fused silica core coated with $85 \mu \mathrm{m}$ polyacrylate (PA).

\subsection{Wine samples}

About twenty-four wine samples were considered in this study for testing the applicability of the proposed methodology. The wines analyzed are fortified wines, namely Madeira wine, with different ageing periods. We considered both young and aged wines: about ten not commercialized wines with less than three years of ageing; nine wines with ageing times comprised between three and five years (these two groups regard young wines); three wines with five up to thirty years old; four wine samples having more than thirty years old (these last groups are relative to aged wines).

\subsection{Extraction procedure}

All extraction experiments were carried out in a TriPlus autosampler, in SPME mode. The factors considered in the optimization phase are presented in Table 1. Also, presented in this table are the minimum and maximum values of their corresponding levels and their data class (categorical or quantitative). The levels to be tested were chosen after a preliminary screening of the relevant information available in the technical literature. The fibers selected were DVB/Car/PDMS and PA, which are the fibers most often considered in the analysis of VFA in food matrixes [5,54-61]. The pre-incubation and extraction time levels were established based on a previous study as well as to ensure that the total extraction time was reasonable for standard working conditions (i.e., not exceeding $60 \mathrm{~min}$ ). Concerning the extraction temperature, the minimum value tested was $40^{\circ} \mathrm{C}$ (fixed according to the equipment capabilities) and the maximum was set to $55^{\circ} \mathrm{C}$. The ethanol influence was tested
Table 1

Experimental factors and the associated levels, considered in the optimization of the HS-SPME extraction performance.

\begin{tabular}{|c|c|c|}
\hline Factor & $\begin{array}{l}\text { Qualitative/ } \\
\text { Quantitative }\end{array}$ & Levels \\
\hline Type of Fiber & Categorical & $\begin{array}{l}\{\mathrm{L} 1-\mathrm{PA}, \mathrm{L} 2-\mathrm{DVB} / \mathrm{Car} / \\
\text { PDMS }\}\end{array}$ \\
\hline Sample volume (ml) & Categorical $^{\mathrm{a}}$ & $\{5,10\}$ \\
\hline Pre-incubation time (min) & Quantitative & {$[0,10]$} \\
\hline Extraction time (min) & Quantitative & {$[15,40]$} \\
\hline $\begin{array}{l}\text { Extraction temperature } \\
\quad\left({ }^{\circ} \mathrm{C}\right)\end{array}$ & Quantitative & {$[40,55]$} \\
\hline Agitation & Categorical & $\{$ L1-Yes, L2-No $\}$ \\
\hline Sample dilution (\%) & Quantitative & {$[4.5,18]$} \\
\hline
\end{tabular}

${ }^{\text {a }}$ Sample volume was considered as a 2-level categorical factor, for practical reasons in terms of sample preparation.

varying its content from $4.5 \%$ up to $18 \%$. The $18 \%$ upper limit was established taking into account that this case study addresses fortified wines, namely the Madeira wine, in which the typical ethanol content is $18 \%$ [62]. Concerning agitation, the analysis evaluates if it is beneficial or not necessary (agitation at $100 \mathrm{rpm}$ during the extraction time).

\subsection{VFA quantification}

Chromatographic analysis was carried out using a GC-MS system, the TRACE GC Ultra gas chromatograph coupled with an ISQ single quadrupole from Thermo Scientific (Hudson, NH, USA), using a DB-WAX $60 \mathrm{~m}$ $\mathrm{x} 0.250 \mathrm{x}$ with $0.50 \mu \mathrm{m}$ film Thickness from Agilent (Folson, CA, USA). The carrier gas was helium at a constant flow rate of $1 \mathrm{~mL} \mathrm{~min}^{-1}$. The injector port was kept at $240^{\circ} \mathrm{C}$, in splitless mode, while the transfer line and the ion source were maintained at $240{ }^{\circ} \mathrm{C}$. The oven temperature program start at $100^{\circ} \mathrm{C}$, holds for $3 \mathrm{~min}$ and increased up to $240^{\circ} \mathrm{C}$ at $5^{\circ} \mathrm{C} \mathrm{min}^{-1}$, being maintained at $240^{\circ} \mathrm{C}$ for $15 \mathrm{~min}$. The total $\mathrm{GC}$ run time was of $45 \mathrm{~min}$. The analysis was carried out in ion monitoring (SIM) operating mode, considering the characteristic and major ions of each analyte, as described in Table 2 . In this table, it is also presented the Kovats indexes for each compound analyzed. The Kovats indexes (KI) were determined analysing an alkane solution (C7 - C30) under the same chromatographic conditions. The mass spectrometer was operated in electron impact (EI) mode at $70 \mathrm{eV}$.

\subsection{Validation of the analytical methodology}

After defining the optimal experimental conditions to carry out HSSPME/GC-MS for the quantification of VFA (see details in Section 4), the analytical methodology was fully validated in terms of linearity, sensitivity, selectivity, precision and accuracy. The calibration curve $(n=7)$ was established following a weighted least-squares regression (WLS) of the area ratio of each VFA-IS onto the corresponding concentration. WLS overcomes the limitations imposed by the assumption of homoscedasticity in Ordinary Least Squares (OLS), which is usually not

\section{Table 2}

Kovats indexes and the characteristic ions of for analytes $(\mathrm{m} / \mathrm{z})$ used for identification and quantification (in bold).

\begin{tabular}{lll}
\hline Compounds & KI & Qualification Ions $(m / z)$ \\
\hline 3- Octanol (PI) & 1418 & $41+55+59+83+101$ \\
Isobutyric acid & 1611 & $41+\mathbf{4 3}+73+88$ \\
Butyric acid & 1676 & $45+\mathbf{6 0}+73$ \\
Isovaleric acid & 1709 & $43+\mathbf{6 0 + 8 7}$ \\
Valeric acid & 1745 & $\mathbf{6 0}+73$ \\
Hexanoic acid & 1795 & $\mathbf{6 0}+73$ \\
Octanoic acid & 2101 & $\mathbf{6 0}+73+101+115$ \\
Nonanoic acid & 2208 & $\mathbf{6 0}+73+129$ \\
Decanoic acid & 2313 & $\mathbf{6 0}+73+129$ \\
Dodecanoic acid & 2521 & $\mathbf{6 0}+73+129+157$ \\
\hline
\end{tabular}


met in analytical data. In this context, WLS provides unbiased estimates for the calibration parameters and more accurate predictions $[63,64]$. In fact, the errors affecting the analytical response are not independent of the analyte concentration, in the sense that the associated variance/uncertainty changes with the level (heteroscedasticity). Therefore, a weighted approached should be followed in order to accommodate the different quality of the measurements when the calibration curve is built $[64,65]$. Furthermore, WLS also provides a better solution to the problem of quantifying the detection limit, namely when it is obtained from the residual standard deviation of the regression curve [66]. The weighted regression was performed considering as the weighting factors of the area ratio, $w_{i}$, the standard deviations estimated from the replicates at each concentration value, $y_{i}$. The Limit of Detection (LOD) and Limit of Quantification (LOQ) were estimated based on the following equations:

$$
\begin{aligned}
& L O D=3.3 \times \frac{b_{w}}{s_{(y / x)_{w}}} \\
& L O Q=10 \times \frac{b_{w}}{s_{(y / x)_{w}}}
\end{aligned}
$$

where, $b_{w}$ is the slope of regression line and $s_{(y / x)_{w}}$ stands for the standard deviation of the regression, calculated as follows,

$s_{(y / x)_{w}}=\left[\frac{\sum_{i} w_{i}\left(y_{i}-\widehat{y}_{i}\right)^{2}}{n-2}\right]^{1 / 2}$

The selectivity was confirmed by the absence of chromatographic interferences at the retention times of each VFA in synthetic wine with $18 \%$ of ethanol. The method's precision was evaluated by inter- and intraday analysis of 2 standard solutions (about 0.5 and $2 \mathrm{mg} \mathrm{L}^{-1}$ ) and a wine sample. The repeatability (intra-day) was determined by the quantification of 10 successive replicates of each standard solution and the wine sample and reproducibility (inter-day) was assessed by performing the same analysis in 3 different days over a week. The values are expressed in terms of relative standard deviation.

A recovery study was also carried out to determine the method's accuracy. A selected wine was spiked, in triplicate, with known amounts each VFA covering 3 different concentrations levels of the calibration range $\left(1,2\right.$ and $\left.4 \mathrm{mg} \mathrm{L}^{-1}\right)$. The recovery was evaluated by computing the percentage of variation between the expected theoretical concentrations, in each level, and the mean values obtained for the concentrations by applying the proposed methodology.

Finally, carry-over was tested by running a blank sample after extracting the highest concentrated working standard solution $\left(16 \mathrm{mg} \mathrm{L}^{-1}\right)$.

\section{Design of Experiments and data analysis}

Once established the factors to be considered and their associated levels (see Table 1), a DSD approach was adopted to define the appropriate testing conditions in each experimental run (treatments). As referred in the introduction, Definitive Screening Designs is a particular class of three levels screening designs (for the quantitative factors), with the capability to provide estimates of the main effects that are unbiased or unconfounded regarding all second-order interactions and among themselves. These designs require a low number of runs, one more than twice the number factors. For more than six factors, they also have the potential ability to estimate the full quadratic model, equation (4), in any possible combination of three factors, a feature that is absent in all other conventional screening designs like the factorial, fraction factorial or Placket-Burman designs.

$y_{i}=\beta_{0}+\sum_{j=1}^{m} \beta_{j} x_{i, j}+\sum_{j=1}^{m-1} \sum_{k=j+1}^{m} \beta_{j k} x_{i, j} x_{i, k}+\sum_{j=1}^{m} \beta_{j j} x_{i, j}^{2}+\varepsilon_{i}$

where $y_{i}$ is the response for $i$ th observation; $x_{i, j}$ the level of factor $j$ in the $i$ th observation; $\beta_{0}, \ldots, \beta_{m, m}$ are unknown constants - many of them being equal to zero according to the sparsity principle; and $\varepsilon_{i}$ is the residual term, which is assumed to be a random variable following an i.i.d. $N\left(0, \sigma^{2}\right)$ distribution.

The ability of estimating quadratic effects steams from the introduction of a center point for one factor in every experimental run plus the addition of a run with all quantitative factors at their center points. For the theoretical details supporting these properties, we refer the interested readers to the relevant technical literature [1-4]. The original version of the DSD was restricted to quantitative factors [67], but a more recent evolution, published in 2013 by the same authors, extended their application to incorporate 2-level categorical factors [2], which allows the application of these designs for addressing the optimization of the factors described in Table 1. Therefore, in this work we have implemented a DSD design for quantitative and 2-level categorical designs, leading to 18 runs with different combinations of factor levels, as described in the design matrix presented in Table 3.

The set of response variables to be simultaneously optimized (maximized in this case), is the analytical response (peak area) corresponding to each VFA under study. The analysis was performed for each VFA individually, as well as for all of them together by modelling their latent variable space.

The analysis in the latent variable space justifies some more discussion, in order to clarify the procedure followed. In a regression setting, the most common modelling frameworks for analyzing data with a latent

Table 3

\begin{tabular}{|c|c|c|c|c|c|c|c|}
\hline Order & Type of fiber & Pre-incub. time (min) & Sample vol. (ml) & Extract. time (min) & Extract. temper. $\left({ }^{\circ} \mathrm{C}\right)$ & Agitation & Sample dilution (\%) \\
\hline 1 & L2 & 0 & L1 & 40 & 40 & L1 & 4.50 \\
\hline 2 & L1 & 5 & L1 & 15 & 40 & L1 & 18 \\
\hline 3 & L2 & 0 & $\mathrm{~L} 2$ & 15 & 55 & L1 & 18 \\
\hline 4 & L2 & 10 & $\mathrm{~L} 2$ & 40 & 40 & $\mathrm{~L} 2$ & 18 \\
\hline 5 & L1 & 0 & L1 & 15 & 55 & L1 & 4.50 \\
\hline 6 & L1 & 0 & $\mathrm{~L} 2$ & 40 & 47.5 & L1 & 18 \\
\hline 7 & $\mathrm{~L} 2$ & 5 & $\mathrm{~L} 2$ & 40 & 55 & $\mathrm{~L} 2$ & 4.50 \\
\hline 8 & L1 & 0 & L1 & 40 & 55 & $\mathrm{~L} 2$ & 9 \\
\hline 9 & L2 & 10 & L1 & 40 & 55 & L1 & 18 \\
\hline 10 & L1 & 5 & L1 & 27.5 & 47.5 & L1 & 9 \\
\hline 11 & L2 & 5 & L2 & 27.5 & 47.5 & L2 & 9 \\
\hline 12 & L1 & 10 & L2 & 15 & 55 & L2 & 18 \\
\hline 13 & L2 & 0 & L1 & 27.5 & 40 & L2 & 18 \\
\hline 14 & L2 & 10 & L2 & 15 & 40 & L1 & 9 \\
\hline 15 & L1 & 0 & L2 & 15 & 40 & L2 & 4.50 \\
\hline 16 & L1 & 10 & L2 & 27.5 & 55 & L1 & 4.50 \\
\hline 17 & L2 & 10 & L1 & 15 & 47.5 & L2 & 4.50 \\
\hline 18 & L1 & 10 & L1 & 40 & 40 & L2 & 4.50 \\
\hline
\end{tabular}

Design matrix obtained from the application of DSD to the factor settings presented in Table 1. (For the coding used in the levels L1 and L2, see Table 1). 
Table 4

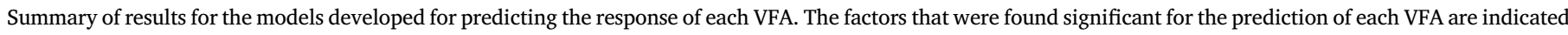

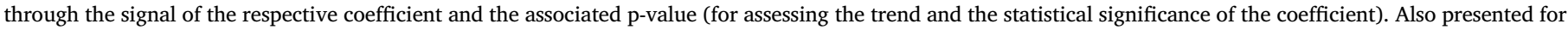
each model is the coefficient of determination $\left(R^{2}\right)$ and the adjusted coefficient of determination $\left(R^{2}{ }_{a d j}\right)$.

\begin{tabular}{|c|c|c|c|c|c|c|c|c|c|}
\hline Factors & iso-C4 & $\mathrm{C} 4$ & iso-C5 & $\mathrm{C} 5$ & $\mathrm{C} 6$ & $\mathrm{C} 8$ & C9 & $\mathrm{C} 10$ & $\mathrm{C} 12$ \\
\hline Fiber (F) & & & $\begin{array}{l}{[-]} \\
(p=0.0321)\end{array}$ & $\begin{array}{l}{[-]} \\
(p=0.0048)\end{array}$ & $\begin{array}{l}{[-]} \\
(p=0.0265)\end{array}$ & & & & \\
\hline \multicolumn{10}{|l|}{$\begin{array}{l}\text { Pre-Incubation } \\
\text { Time (PIT) }\end{array}$} \\
\hline $\begin{array}{l}\text { Sample Volume } \\
\text { (VOL) }\end{array}$ & & & $\begin{array}{l}{[-]} \\
(p=0.0356)\end{array}$ & $\begin{array}{l}{[-]} \\
(p=0.0205)\end{array}$ & & & & & $\begin{array}{l}{[-]} \\
(p=0.0113)\end{array}$ \\
\hline $\begin{array}{l}\text { Extraction Time } \\
\text { (ETI) }\end{array}$ & & & $\begin{array}{l}{[+]} \\
(p=0.0058)\end{array}$ & $\begin{array}{l}{[+]} \\
(p=0.0017)\end{array}$ & $\begin{array}{l}{[+]} \\
(p=0.0186)\end{array}$ & $\begin{array}{l}{[+]} \\
(\mathrm{p}=0.0201)\end{array}$ & $\begin{array}{l}{[+]} \\
(\mathrm{p}=0.0172)\end{array}$ & & $\begin{array}{l}{[+]} \\
(\mathrm{p}=0.0047)\end{array}$ \\
\hline \multicolumn{10}{|l|}{$\begin{array}{l}\text { Temperature } \\
\text { (ETE) }\end{array}$} \\
\hline \multicolumn{10}{|l|}{ Agitation (A) } \\
\hline $\begin{array}{l}\text { Sample Dilution } \\
\text { (DIL) }\end{array}$ & $\begin{array}{l}{[-]} \\
(p<0.0001)\end{array}$ & $\begin{array}{l}{[-]} \\
(p<0.0001)\end{array}$ & $\begin{array}{l}{[-]} \\
(p<0.0001)\end{array}$ & $\begin{array}{l}{[-]} \\
(p<0.0001)\end{array}$ & $\begin{array}{l}{[-]} \\
(p=0.0004)\end{array}$ & $\begin{array}{l}{[-]} \\
(p=0.0132)\end{array}$ & $\begin{array}{l}{[-]} \\
(p=0.0126)\end{array}$ & $\begin{array}{l}{[-]} \\
(p=0.0162)\end{array}$ & $\begin{array}{l}{[-]} \\
(p<0.0001)\end{array}$ \\
\hline DIL*ETE & & $\begin{array}{l}{[+]} \\
(p=0.0114)\end{array}$ & & & & & & & \\
\hline DIL*DIL & & & $\begin{array}{l}{[+]} \\
(\mathrm{p}=0.0258)\end{array}$ & $\begin{array}{l}{[+]} \\
(p=0.0008)\end{array}$ & & & & & \\
\hline VOL*ETI & $\begin{array}{l}{[-]} \\
(p=0.0191)\end{array}$ & & $\begin{array}{l}{[-]} \\
(p=0.0132)\end{array}$ & & & & & & \\
\hline $\mathrm{F}^{*} \mathrm{VOL}$ & & & & $\begin{array}{l}{[+]} \\
(p=0.0123)\end{array}$ & & & & & \\
\hline $\mathbf{R}^{2}$ & 0.83 & 0.75 & 0.95 & 0.95 & 0.74 & 0.49 & 0.50 & 0.31 & 0.74 \\
\hline $\mathbf{R}^{2} \mathbf{a d j}$ & 0.81 & 0.72 & 0.92 & 0.92 & 0.69 & 0.43 & 0.44 & 0.27 & 0.68 \\
\hline
\end{tabular}

structure are Principal Component Regression (PCR) and Partial Least Squares (PLS). The implicit assumption of the latent variable model for which PCR and PLS provide estimates, is that the variability of all variables to be modeled, both in the X-block and in Y-block, is driven by a set of unobservable variation sources (the latent variables). However, in the present case this does not happen. Being a DOE scenario, the X-block does not present a latent structure (the drivers of variability are the design factors and these are obviously observable), and only the Y-block present a latent structure. In this context, the actual model structure to be inferred and analyzed is the following one (see more in Ref. [68]):

$\mathbf{Y}=\mathbf{t} \cdot \mathbf{P}^{T}+\boldsymbol{\varepsilon}_{2}, \boldsymbol{\varepsilon}_{2} \sim$ i.i.d. $N_{m}\left(0_{m}, \boldsymbol{\Sigma}_{\varepsilon_{2}}\right)$

$\mathbf{t}=\mathbf{X} \cdot \mathbf{B}+\boldsymbol{\varepsilon}_{1}, \boldsymbol{\varepsilon}_{1} \sim$ i.i.d. $N_{a}\left(0, \boldsymbol{\Lambda}_{\varepsilon_{1}}\right)$

where, $\mathbf{t}$ and $\mathbf{P}$ are the PCA scores and the loading matrix for the responses (Y-block), respectively. The response scores are regressed onto the experimental factors, the X-block, which does not have a latent structure. $\varepsilon_{1}$ stands for the regression residuals with covariance $\boldsymbol{\Lambda}_{\varepsilon_{1}}$ (a diagonal matrix), and $\varepsilon_{2}$ represents the reconstruction error of the responses, modeled as an i.i.d. $N_{m}$ process ( $m$ being the number of output variables considered), with zero mean and covariance $\boldsymbol{\Sigma}_{\varepsilon_{2}}$. The estimation of the model structure (6) involves two stages: (i) conduct a PCA analysis of the responses and retain the scores that explain the relevant variability for the analysis; (ii) regress the retained scores onto the $\mathrm{X}$ block, i.e., onto the experimental factors, and obtain the predictive model. This procedure was followed in the next section, where the results of the analysis are reported. The model obtained can then be used to determine the factor levels optimizing the extraction performance, after a preliminary interpretation of the meaning of the scores under analysis (based on the loading coefficients).

The generation of the DSD design matrix and the subsequent data analysis (model estimation and optimization) were conducted in JMPPRO ver. 12.1.0 (64-bit) (SAS Institute Inc.). All extraction experiments

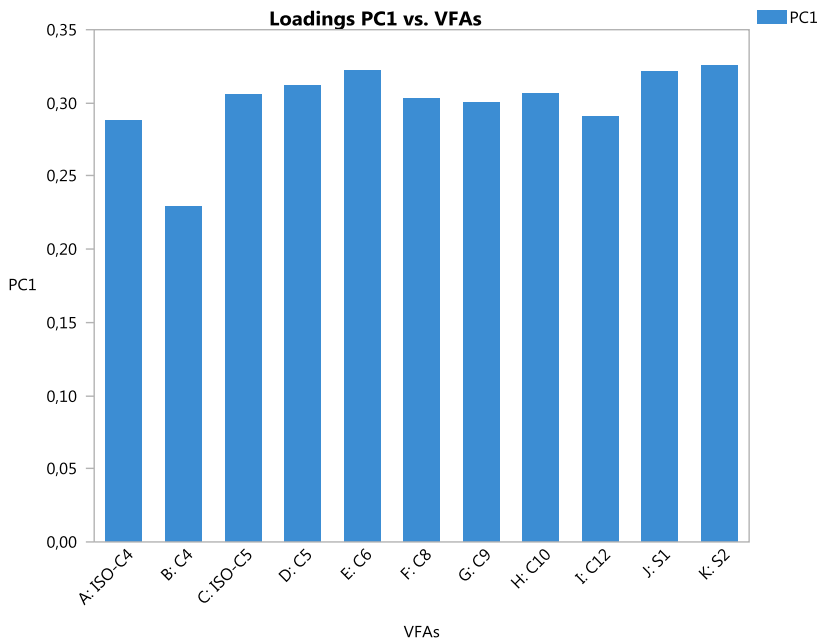

VFAs

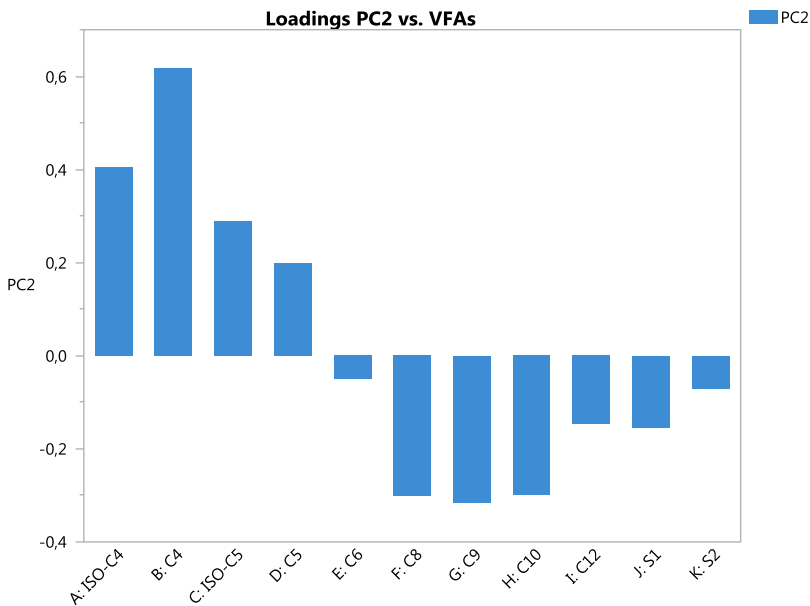

a) b)

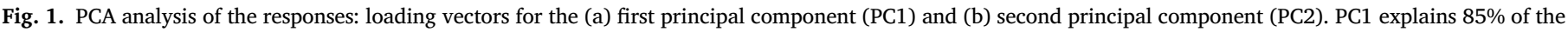
total variability of the responses and PC2 explains $11 \%$. 
were carried out using the same wine matrix, namely a 3 year old Madeira wine sample.

\section{Results and discussion}

A DSD design applied to the factors defined in Table 1 resulted in 18 treatments to be run in random order (the experimental conditions tested are listed in Table 3). All target VFAs were analyzed and the respective response variables recorded, which in this case were given by their chromatographic peak areas.

Data analysis revealed that it was indeed possible to construct individual models with good explanation power for most of the VFAs under analysis ( $R^{2}$ between 0.75 and 0.95 for 6 out of 9 VFAs). Even for the cases where the model's quality of fit was found not to be so high, they still present several statistically significant model parameters (at a significance level of 5\%) which allowed carrying out the optimization task towards the maximization of the peak area. Table 4 summarizes the modelling results obtained for each compound in this first stage of data analysis, dedicated to model building for each individual analyte. In order to maintain the depicted information at a manageable level we just report the signal of the coefficients retained for each model and the associated p-values, to highlight that no opposing trends were found between the different VFAs and the factors that actively contribute to their variation. Therefore, in this case the optimal extraction conditions should be valid for all VFAs, with no need to establish compromises in the factor levels during the simultaneous analysis of all responses.

The correlation structure of the responses (the chromatographic responses of the different VFAs, i.e., their peak area) was also carefully analyzed in the scope of the more complete and rigorous treatment based on the model structure presented in equation (5). This process consists of two steps: (i) PCA analysis and selection of the scores to be retained; (ii) regression of the relevant scores onto the experimental factors and model analysis (see Section 4). Regarding the first step, a PCA analysis on the auto-scaled variables (i.e., variables mean centered and scaled to unit variance; this analysis is equivalent to perform the eigen-decomposition of the correlation matrix) reveals a strong latent structure of the responses, with PC1 representing $85 \%$ of the total variability, while PC2 captures $11 \%$ of the total variability. Fig. 1 presents the loading vectors for these principal components. It is clear that all VFA signals are positively correlated, and PC1 represents a sort of average of their chromatographic responses. PC2, on the other hand, represents a contrast between analytes with shorter chain lengths and that are more volatile, and those with longer chain lengths and less volatile.

The PCA component that is more relevant for describing the underlying structure of the chromatographic responses of all VFAs is clearly PC1, given its relative importance (explanation power of the Yblock variability) in comparison with the others - the first component explains $85 \%$ of the overall Y-variability. Therefore, in step (ii) a model was derived for predicting the scores of PC1. Table 5 summarizes the results obtained in this model (again, the signal of the coefficients and their p-values are reported). Comparing the results obtained in Table 4 regarding the individual analysis of the VFAs, with those in Table 5 for the analysis of their underlying latent structure (PC1), one can verify that all coefficients that were found to be significant in the univariate and multivariate models (regarding main effects and their interactions), do present the same trends. Therefore no trade-off analysis is required to be carried out. The factor settings that maximize the chromatographic area of each individual VFA are entirely coherent with those that maximize the scores of PC1 (note that all the loadings have positive values for all VFA's, therefore the optimum corresponds to maximizing PC1; otherwise, if the signals of the loadings were negative, a minimization of the model for this principal component should be carried out instead).

Finally, the optimal (consensus) conditions were found by
Table 5

Summary of results for the models developed for predicting the scores of the first two principal components. The factors that were found significant for the prediction of each principal component scores' are indicated through the signal of the respective coefficient and the associated p-value (for assessing the trend and the associated statistical significance of the coefficient). Also presented for each model is the coefficient of determination $\left(R^{2}\right)$ and the adjusted coefficient of determination $\left(R^{2}{ }_{a d j}\right)$.

\begin{tabular}{ll}
\hline Factors & PC1 \\
\hline Fiber (F) & \\
Pre-Incubation Time (PIT) & {$[-](\mathrm{p}=0.0417)$} \\
Sample Volume (SV) & {$[+](\mathrm{p}=0,0029)$} \\
Extraction Time (ETI) & \\
Extraction Temperature (ETE) & {$[-](\mathrm{p}<0.0001)$} \\
Agitation (A) & \\
Sample Dilution (DIL) & {$[+](\mathrm{p}=0.0699)$} \\
DIL*ETE & \\
DIL*DIL & \\
VOL*ETI & 0.83 \\
$\mathrm{~F}^{*}$ VOL & 0.77 \\
\hline $\mathbf{R}^{2}$ & \\
$\mathbf{R}^{2} \mathbf{a d j}$ &
\end{tabular}

Table 6

Optimal levels for the experimental factors optimizing HS-SPME performance.

\begin{tabular}{ll}
\hline Factor & Qualitative/Quantitative \\
\hline Type of Fiber & L2- DVB/Car/PDMS \\
Sample volume & $10 \mathrm{~mL}$ \\
Pre-incubation time & $0 \mathrm{~min}$ \\
Extraction time & $40 \mathrm{~min}$ \\
Extraction temperature & $40^{\circ} \mathrm{C}$ \\
Agitation & $\mathrm{L} 2-\mathrm{No}$ \\
Sample Dilution & $18 \%$ \\
\hline
\end{tabular}

optimizing the significant factors in the models, while the others were set to the conditions that are more convenient, inexpensive or imply less experimental effort. The optimal solution is presented in Table 6 .

Sample volume, content of ethanol (sample dilution) and extraction time, are the factors with more significant impact in the extraction performance. The ethanol content (sample dilution) can influence the extraction of target analytes since the ethanol molecules can compete for the actives sites of the fibre, remaining fewer to retain the target analytes. To overcome this effect, sample dilution was tested to verify if a lower percentage of ethanol can lead to better extraction efficiencies. In line with what was reported in Ref. [5], our results indicate that using an ethanol content of $18 \%$ is more advantageous than applying sample dilution. This factor was found to be highly significant, both considering the individual responses and the latent variable model. The second factor with a significant impact on the responses was the extraction time. The analysis revealed the importance of keeping it at its high level, namely 40min (even though no influence was signalled for some VFAs, such as the isobutyric, butyric and decanoic acids). Concerning the sample volume, the results suggest that it should be kept at $10 \mathrm{~mL}$ in a vial with $20 \mathrm{~mL}$ of capacity; the extraction is not favoured when smaller quantities of the sample are used. The remaining factors were not found to be so relevant, and therefore their settings were established based on the results of the individual VFA chromatographic areas, since there were no situations requiring a compromise due to opposite trends in the factors influence. The selected fibre was DVB/Car/PDMS, a bipolar fibre which is adequate for the more polar compounds (butyric, isobutyric, valeric and isovaleric acids) and for less polar compounds (acids with a number of carbons from six up to twelve). Regarding the incubation time, the extraction temperature and agitation, results indicate that these factors do not present significant impact on the responses. 


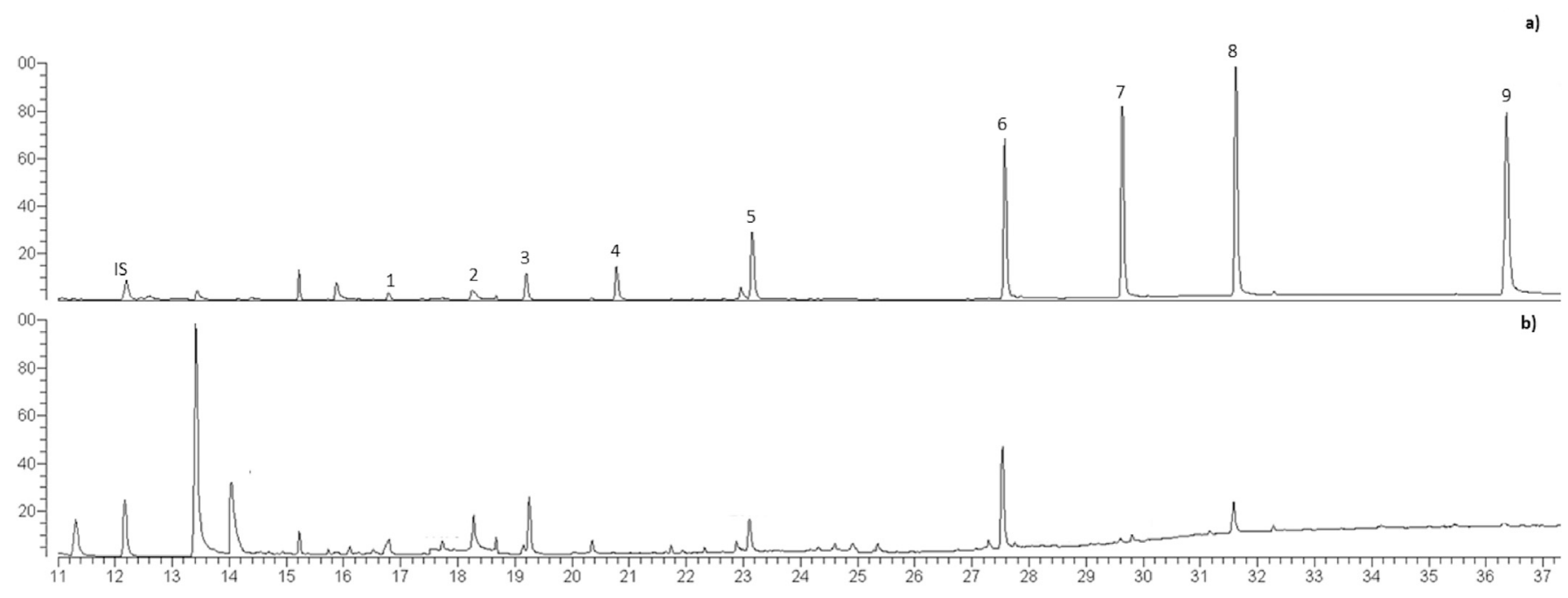

Fig. 2. Chromatograms of a $1 \mathrm{mg} \mathrm{L}^{-1}$ standard solution (a) and an aged wine (b). IS- Internal standard, 1 - isobutyric acid, 2 - butyric acid, 3- isovaleric acid, 4 - valeric acid, 5 - hexanoic acid, 6 - octanoic acid, 7 - nonanoic acid, 8 - decanoic acid, 9 - dodecanoic acid.

Table 7

Figures of merit for the HS-SPME/GC-MS methodology to quantify VFA.

\begin{tabular}{|c|c|c|c|c|c|c|c|c|}
\hline Factor & Slope, $b_{w}$ & Intercept, $a_{w}$ & $R_{w}^{2}$ & LOD (mg. $\left.\mathrm{L}^{-1}\right)$ & LOQ (mg. $\left.\mathrm{L}^{-1}\right)$ & Recovery (\%) & Repeatability (\%) & Reproducibility (\%) \\
\hline Isobutyric acid & 0.187 & 0.031 & 0.999 & 0.010 & 0.031 & 103.50 & 6.59 & 8.07 \\
\hline Butyric acid & 0.257 & 0.014 & 0.999 & 0.005 & 0.014 & 100.26 & 7.21 & 8.61 \\
\hline Isovaleric acid & 0.529 & 0.024 & 0.999 & 0.002 & 0.007 & 108.42 & 4.86 & 7.48 \\
\hline Valeric acid & 0.625 & -0.032 & 0.999 & 0.017 & 0.052 & 101.70 & 5.93 & 5.56 \\
\hline Hexanoic acid & 1.201 & -0.140 & 0.999 & 0.032 & 0.096 & 102.50 & 4.43 & 5.34 \\
\hline Octanoic acid & 1.909 & -0.224 & 0.999 & 0.023 & 0.069 & 100.39 & 2.96 & 4.56 \\
\hline Nonanoic acid & 2.086 & -0.262 & 0.999 & 0.015 & 0.046 & 103.82 & 4.20 & 6.43 \\
\hline Decanoic acid & 2.225 & -0.270 & 0.999 & 0.029 & 0.089 & 104.27 & 3.98 & 5.64 \\
\hline Dodecanoic acid & 2.476 & -0.239 & 0.999 & 0.029 & 0.087 & 103.92 & 6.54 & 6.94 \\
\hline
\end{tabular}

\section{Method validation and test in new wine samples}

In this section we report the results of the validation study carried out for the operation of the HS-SPME extraction at the optimal conditions, as well as its application to the analysis of new wine samples.

\subsection{Validation of the optimal extraction conditions}

The selectivity of the methodology was confirmed by the absence of interferents at the retention times associated to each VFA in the calibration solutions, as well as the absence of any coeluted compounds with target compounds when wine was analyzed under the established conditions. In Fig. 2, it is presented a typical chromatogram of a standard solution and also an example of a wine sample.

The figures of merit adopted for validating the extraction protocol are summarized in Table 7. All the regression curves were found to exhibit good scores in terms of linearity, $R_{w}^{2}$. The comparison of the obtained LOD and LOQ with those reported in literature is not straightforward, due to the well-known difference between the methods used to estimate these parameters [69,70]. In the present study, LOD and LOQ were estimated based on the standard deviation of the weight regression line and its slope, equations (1) and (2). This procedure is known to lead to higher values for these parameters [71]. Nonetheless, the LOD and LOQ obtained are closer or even lower than those found in the literature when other approaches are followed [72-74], and for several times higher than the odor threshold of each VFA [75], confirming that the proposed methodology has an adequate sensitivity to the intended analysis. The accuracy results, which were obtained from a recovery study at three concentrations, also confirm the method's accuracy, with values ranging from $100.26 \%$ for butyric acid up to $108.42 \%$ for isovaleric acid. Good results were also obtained in terms of repeatability and reproducibility, all lower than $9 \%$.

\subsection{Application of the optimized HS-SPME to Madeira fortified wines}

The optimized HS-SPME methodology was finally applied to the quantification of VFAs in a variety of samples of Madeira fortified wines. The focus on this class of compounds relies on their influence in the wine aroma, a consequence of their low olfaction thresholds and their volatility at room temperature. When presented at, or below, the odor thresholds, these compounds contribute to the complexity of the wine bouquet. However, above the olfactory threshold they can have a detrimental influence on wine aroma. In the study reported in Ref. [76] concerning the identification of potential odorant compounds in aged Madeira wines, it was found that there were seven volatile fatty acids present above their odor thresholds in aged wines and four of them, namely the isovaleric, butyric, decanoic, hexanoic acids, were in the top of the list of the main active compounds in these wines. In the present study, we consider the application of the methodology developed for VFA quantification to a relatively high number of samples, covering young and aged Madeira wines. Table 8 presents the concentrations of each VFA obtained in the present study, normalized by their corresponding threshold values (the odor activity value (OAV)), to facilitate the identification of their importance as odor active compounds in Madeira wines. According to our results and in line with the study mentioned above, isovaleric still remains the VFA presenting more influence within this class of compounds, followed by butyric and isobutyric acid. Also, the order of magnitude of OAV is similar to that found previously by 
Table 8

Odor activity values (OAV) obtained from the application of the optimized HS-SPME/GC-MS methodology.

\begin{tabular}{|c|c|c|c|c|c|c|c|c|c|}
\hline Wine samples & $\begin{array}{l}\text { Isobutyric } \\
\text { acid }\end{array}$ & $\begin{array}{l}\text { Butyric } \\
\text { acid }\end{array}$ & $\begin{array}{l}\text { Isovaleric } \\
\text { acid }\end{array}$ & $\begin{array}{l}\text { Valeric acid } \\
\text { a }\end{array}$ & $\begin{array}{l}\text { Hexanoic } \\
\text { acid }\end{array}$ & $\begin{array}{l}\text { Octanoic } \\
\text { acid }\end{array}$ & $\begin{array}{l}\text { Nonanoic } \\
\text { acid }\end{array}$ & $\begin{array}{l}\text { Decanoic } \\
\text { acid }\end{array}$ & $\begin{array}{l}\text { Dodecanoic } \\
\text { acid }\end{array}$ \\
\hline $\begin{array}{l}\text { Odor threshold } \\
\left(\mathrm{mgL}^{-1}\right)^{\mathrm{b}}\end{array}$ & 2.300 & 0.173 & 0.033 & - & 0.420 & 0.500 & 3.000 & 1.000 & 6.100 \\
\hline \multirow[t]{10}{*}{$<3$ years old } & 0.610 & 1.629 & 14.284 & 0.471 & 3.367 & 1.925 & 0.053 & 0.338 & 0.028 \\
\hline & 0.449 & 3.085 & 13.586 & 0.448 & 3.831 & 2.295 & 0.051 & 0.265 & 0.024 \\
\hline & 0.620 & 5.295 & 13.968 & 0.462 & 2.239 & 3.198 & 0.058 & 1.134 & 0.061 \\
\hline & 0.498 & 4.669 & 11.688 & 0.386 & 2.376 & 3.854 & 0.049 & 1.204 & 0.036 \\
\hline & 0.607 & 4.354 & 10.853 & 0.358 & 2.190 & 3.530 & 0.048 & 1.040 & 0.030 \\
\hline & 0.763 & 3.941 & 9.761 & 0.322 & 2.174 & 3.329 & 0.048 & 0.962 & 0.032 \\
\hline & 0.509 & 4.123 & 10.232 & 0.338 & 3.142 & 4.677 & 0.051 & 1.207 & 0.035 \\
\hline & 0.092 & 7.447 & 5.795 & 0.191 & 1.579 & 3.849 & 0.040 & 1.128 & 0.034 \\
\hline & 0.510 & 6.782 & 17.046 & 0.565 & 3.048 & 4.473 & 0.048 & 0.978 & 0.030 \\
\hline & 0.536 & 7.055 & 7.0551 & 0.594 & 3.174 & 4.611 & 0.047 & 0.996 & 0.030 \\
\hline \multirow[t]{9}{*}{$3-5$ years old } & 0.433 & 0.895 & 17.286 & n.q. & 2.042 & 0.760 & 0.048 & 0.160 & 0.020 \\
\hline & 0.472 & 0.840 & 18.402 & n.q. & 2.165 & 0.788 & 0.051 & 0.166 & 0.021 \\
\hline & 1.898 & 0.595 & 16.710 & n.q. & 2.019 & 2.252 & 0.048 & 0.326 & 0.021 \\
\hline & 1.630 & 0.832 & 19.662 & n.q. & 2.113 & 2.418 & 0.052 & 0.330 & 0.022 \\
\hline & 0.265 & 4.693 & 9.585 & n.q. & 2.364 & 1.379 & 0.049 & 0.233 & 0.023 \\
\hline & 0.433 & 2.142 & 16.661 & 0.550 & 1.305 & 0.734 & 0.047 & 0.177 & 0.020 \\
\hline & 0.450 & 2.242 & 16.524 & 0.545 & 1.311 & 0.719 & 0.047 & 0.168 & 0.020 \\
\hline & 0.228 & 1.477 & 15.401 & 0.508 & 2.049 & 1.391 & 0.053 & 0.240 & 0.022 \\
\hline & 0.199 & 0.865 & 13.493 & 0.445 & 1.693 & 1.415 & 0.049 & 0.251 & 0.021 \\
\hline \multirow[t]{3}{*}{ 5-30 years old } & 0.889 & 2.765 & 30.811 & 1.017 & 3.245 & 2.205 & 0.053 & 0.204 & 0.019 \\
\hline & 0.703 & 4.948 & 29.269 & 0.966 & 4.428 & 2.341 & 0.048 & 0.238 & 0.020 \\
\hline & 0.845 & 5.253 & 39.265 & 1.296 & 2.478 & 1.376 & 0.052 & 0.212 & 0.021 \\
\hline \multirow[t]{4}{*}{$>30$ years old } & 1.891 & 22.767 & 51.802 & 1.709 & 2.452 & 1.115 & 0.054 & 0.338 & 0.027 \\
\hline & 1.891 & 22.767 & 51.802 & 1.709 & 2.452 & 1.115 & 0.054 & 0.338 & 0.027 \\
\hline & 5.890 & 45.254 & 75.163 & 2.480 & 3.506 & 1.685 & 0.052 & 0.196 & 0.022 \\
\hline & 3.707 & 23.284 & 59.456 & 1.962 & 2.650 & 1.451 & 0.052 & 0.202 & 0.021 \\
\hline
\end{tabular}

a Concentration values $\left(\mathrm{mg} \mathrm{L}^{-1}\right.$ ), since the odor activity value is not available.

b Campo et al. [80] E. Campo, V. Ferreira, A. Escudero, J.C. Marqués, J. Cacho, Analytica Chimica Acta, 563:1-2 (2006) $180-187$.

Ref. [76]. It should be noted that although these compounds are usually associated with negative aromas in table wines, their presence at concentrations higher then odor threshold in fortified wines do not compromise wine quality. The concentration ranges found in the wines analyzed in this study were similar to those found in other wines using different techniques, with the exception of the long aged wines (more than 30 years of ageing) [5,76-79]. These results reinforce the previously presented validation results in terms of methodology applicability and performance.

\section{Conclusions}

In this article we explore and assess the application of Definitive Screening Design (DSD) and latent variable analysis to derive the optimal extraction conditions of a critical analytical technique for a range of wine analytes: Headspace Solid-Phase MicroExtraction (HS-SPME). The application of DOE methodologies has been growing in importance in the development of new and/or improved analytical methodologies. The associated benefits of this trend include both a reduction in operational costs (less time and resources) as well as granted access to more insights on the phenomena and variability sources in the experimental domain of interest, using a low number of experiments. Definitive Screening Design is a recent DOE approach that offers several interesting features for achieving these purposes, and that may present added value to the activities conducted in analytical laboratories. Among their properties, one can refer the orthogonal estimation of main effects, which are also completely unaliased (or unconfounded) with two factor interactions; pure quadratic effects can also be estimated, if their strength is large enough and if certain reasonable sparsity conditions are met; and of course, the low number of runs involved, which is of the order of twice as many the number of quantitative factors.

In this study, seven HS-SPME parameters where simultaneously evaluated and optimized, in the scope of the quantification of VFA in wines. The optimal conditions found were: fiber type DVB/Car/PDMS fiber, $40 \mathrm{~min}$ of extraction always at $40^{\circ} \mathrm{C}$, considering $10 \mathrm{~mL}$ of sample in a $20 \mathrm{~mL}$ vial without incubation time, agitation and sample dilution. The proposed analytical methodology was fully characterized and validated, showing good results in terms of linearity, sensitivity, selectivity precision and accuracy. Its application to independent samples also demonstrated that it is selective (good peak resolution) and adequate for VFA quantification.

\section{Acknowledgements}

Ana C. Pereira and João M. Leça are thankful to the Agência Regional para o Desenvolvimento da Investigação Tecnologia e Inovação (ARDITI) for the financial support through their Post Doc and $\mathrm{PhD}$ grants in the scope of the project M1420 - 09-5369-FSE-000001.

\section{References}

[1] B. Jones, C.J. Nachtsheim, J. Qual. Technol. 43 (1) (2011) 1-15.

[2] B. Jones, C.J. Nachtsheim, J. Qual. Technol. 45 (1) (2013) 1-9.

[3] B. Jones, Qual. Eng. 28 (1) (2016) 98-106.

[4] B. Jones, C.J. Nachtsheim, Technometrics 58 (1) (2016) 74-83.

[5] S.J. Pérez Olivero, J.P. Pérez Trujillo, Anal. Chim. Acta 696 (1-2) (2011) 59-66.

[6] S. Azzi-Achkouty, N. Estephan, N. Ouaini, D.N. Rutledge, CRC Crit. Rev. Food Sci. 57 (2017) 2009-2020.

[7] E.S. Hecht, A.L. Oberg, D.C. Muddiman, J. Am. Soc. Mass Spectrom. 27 (5) (2016) $767-785$.

[8] P.W. Araujo, R.G. Brereton, TrAC Trends Anal. Chem. (Reference Ed.) 15 (1) (1996) 26-31.

[9] R. Leardi, Anal. Chim. Acta 652 (1-2) (2009) 161-172.

[10] M.A. Bezerra, R.E. Santelli, E.P. Oliveira, L.S. Villar, L.A. Escaleira, Talanta 76 (5) (2008) 965-977.

[11] D.C. Montgomery, G.C. Runger, Design of Experiments with Several Factors, United States of America, 2003.

[12] J. Antony, 3-Understanding Key Interactions in Processes, Butterworth-Heinemann, Oxford, 2003.

[13] T. Lundstedt, E. Seifert, L. Abramo, B. Thelin, Å. Nyström, J. Pettersen, R. Bergman, Chemometr. Intell. Lab. Syst. 42 (1-2) (1998) 3-40. 
[14] M.P. Callao, TrAC Trends Anal. Chem. (Reference Ed.) 62 (2014) 86-92.

[15] A.G. González, Anal. Chim. Acta 360 (1-3) (1998) 227-241.

[16] D.B. Hibbert, J. Chromatogr. B 910 (2012) 2-13.

[17] C.R.T. Tarley, G. Silveira, W.N.L. dos Santos, et al., Microchem. J. 92 (1) (2009) $58-67$.

[18] G. Hanrahan, R. Montes, F.A. Gomez, Anal. Bioanal. Chem. 390 (1) (2008) $169-179$.

[19] D.J. Weston, Analyst 135 (4) (2010) 661-668.

[20] S.L.C. Ferreira, W.N.L. dos Santos, C.M. Quintella, B.c.B. Neto, J.M. Bosque-Sendra, Talanta 63 (4) (2004) 1061-1067.

[21] B. Dejaegher, Y. Vander Heyden, J. Pharmaceut. Biomed. 56 (2) (2011) 141-158.

[22] A.L. Oberg, O. Vitek, J. Proteome Res. 8 (5) (2009) 2144-2156.

[23] S.L.C. Ferreira, R.E. Bruns, H.S. Ferreira, et al., Anal. Chim. Acta 597 (2) (2007) 179-186.

[24] M.I. Cervera, J. Beltran, F.J. Lopez, F. Hernandez, Anal. Chim. Acta 704 (1-2) (2011) 87-97.

[25] M. Fernandez-Alvarez, M. Llompart, J.P. Lamas, M. Lores, C. Garcia-Jares, R. Cela, T. Dagnac, Anal. Chim. Acta 617 (1-2) (2008) 37-50.

[26] E. Gionfriddo, A. Naccarato, G. Sindona, A. Tagarelli, Anal. Chim. Acta 747 (2012) $58-66$.

[27] C.H. Kowalski, G.A.d. Silva, R.J. Poppi, H.T. Godoy, F. Augusto, Anal. Chim. Acta 585 (1) (2007) 66-75.

[28] R. Morales, M. Cruz Ortiz, L.A. Sarabia, Anal. Chim. Acta 754 (2012) 20-30.

[29] J. Salafranca, C. Domeño, C. Fernández, C. Nerín, Anal. Chim. Acta 477 (2) (2003) $257-267$.

[30] Y. Zhang, J. Zhang, Anal. Chim. Acta 627 (2) (2008) 212-218.

[31] G. Basaglia, L. Pasti, M.C. Pietrogrande, Anal. Bioanal. Chem. 399 (6) (2011) 2257-2265.

[32] F. Lestremau, F.A.T. Andersson, V. Desauziers, J.-L. Fanlo, Anal. Chem. 75 (11) (2003) 2626-2632.

[33] L.F. Cuevas-Glory, O. Sosa-Moguel, J. Pino, E. Sauri-Duch, Food Anal. Meth. 8 (4) (2015) 1005-1013.

[34] J.N. Bianchin, G. Nardini, J. Merib, A.N. Dias, E. Martendal, E. Carasek, Food Chem. 145 (2014) 1061-1065.

[35] K.W. Cheong, C.P. Tan, H. Mirhosseini, et al., Food Chem. 125 (4) (2011) 1481-1489.

[36] A. Melo, A. Aguiar, C. Mansilha, O. Pinho, I.M.P.L.V.O. Ferreira, Food Chem. 130 (4) (2012) 1090-1097.

[37] T. Pérez-Palacios, C. Petisca, A. Melo, I.M.P.L.V.O. Ferreira, Food Chem. 135 (3) (2012) 1337-1343.

[38] M. Petrozziello, D. Borsa, M. Guaita, V. Gerbi, A. Bosso, Food Chem. 135 (4) (2012) 2483-2489.

[39] R. Castro Mejías, R. Natera Marín, M. de Valme García Moreno, C. García Barroso, J. Chromatogr. a 953 (1-2) (2002) 7-15.

[40] R. Castro Mejías, R. Natera Marín, M. de Valme García Moreno, C. García Barroso, J. Chromatogr. a 995 (1-2) (2003) 11-20.

[41] B. Cavaliere, M. Monteleone, A. Naccarato, G. Sindona, A. Tagarelli, J. Chromatogr. a 1257 (2012) 149-157.

[42] J. Dron, R. Garcia, E. Millán, J. Chromatogr. a 963 (1-2) (2002) 259-264.

[43] A. Gaujac, E.S. Emídio, S. Navickiene, S.L.C. Ferreira, H.S. Dórea, J. Chromatogr. a 1203 (1) (2008) 99-104.

[44] S. Legrand, J. Dugay, J. Vial, J. Chromatogr. a 999 (1-2) (2003) 195-201.

[45] C. Qiu, M. Cai, J. Chromatogr. a 1217 (8) (2010) 1191-1202.

[46] S. Schramm, D. Vailhen, M.C. Bridoux, J. Chromatogr. a 1433 (2016) 24-33.
[47] J.M. Leça, A.C. Pereira, A.C. Vieira, M.S. Reis, J.C. Marques, Anal. Chim. Acta 887 (2015) 101-110.

[48] D. Arroyo, M.C. Ortiz, L.A. Sarabia, J. Chromatogr. a 1218 (28) (2011) 4487-4497.

[49] C. Coscollà, S. Navarro-Olivares, P. Martí, V. Yusà, Talanta 119 (2014) 544-552.

[50] E. Passeport, A. Guenne, T. Culhaoglu, S. Moreau, J.-M. Bouyé, J. Tournebize, J. Chromatogr. a 1217 (33) (2010) 5317-5327.

[51] L.B. Abdulra'uf, G.H. Tan, Food Chem. 177 (2015) 267-273.

[52] I. Calejo, N. Moreira, A.M. Araújo, M. Carvalho, M.d.L. Bastos, P.G. de Pinho, Talanta 148 (2016) 486-493.

[53] I. Stamati, S. Akkermans, F. Logist, E. Noriega, J. Van Impe, Food Res. Int. 89 (1) (2016) 689-700.

[54] M. Ziino, C. Condurso, V. Romeo, D. Giuffrida, A. Verzera, Int. Dairy J. 15 (6-9) (2005) 585-593.

[55] D. Wang, J. Cai, B.-Q. Zhu, G.-F. Wu, C.-Q. Duan, G. Chen, Y. Shi, Food Chem. 177 (2015) 346-353.

[56] A.F. González-Córdova, B. Vallejo-Cordoba, J. Agric. Food Chem. 49 (10) (2001) 4603-4608.

[57] I. Romero, D.L. García-González, R. Aparicio-Ruiz, M.T. Morales, Talanta 134 (2015) 394-401.

[58] M.A. Farag, D.M. Rasheed, I.M. Kamal, Food Res. Int. 78 (2015) 327-335.

[59] F.K. Tavaria, A.C. Silva Ferreira, F.X. Malcata, J. Dairy Sci. 87 (12) (2004) $4064-4072$.

[60] O. Pinho, I.M.P.L.V.O. Ferreira, M.A. Ferreira, Anal. Chem. 74 (2002) 5199-5204.

[61] T.L. Felicio, E.A. Esmerino, V.A.S. Vidal, et al., Food Chem. 196 (2016) 628-637.

[62] A.C. Pereira, M.J. Carvalho, A. Miranda, et al., Chemometr. Intell. Lab. Syst. 154 (2016) 176-184.

[63] E. Santoyo, S.P. Verma, J. Chromatogr. a 997 (1-2) (2003) 171-182.

[64] B. Brasil, R.J.N. Bettencourt da Silva, M.F.G.F.C. Camões, P.A.S. Salgueiro, Anal. Chim. Acta 804 (2013) 287-295.

[65] J.N. Miller, J.C. Miller, Calibration Methods in Instrumental Analysis: Regression and Correlation, Prentice Hall/Pearson, England, 2010.

[66] J. Vial, A. Jardy, Anal. Chem. 71 (14) (1999) 2672-2677.

[67] B. Jones, C.J. Nachtsheim, J. Qual. Technol. 43 (1) (2011) 1-15.

[68] M.S. Reis, P.M. Saraiva, Ind. Eng. Chem. Res. 51 (2012), 4524-4266.

[69] R. Ismail, H.Y. Lee, N.A. Mahyudin, F. Abu Bakar, J. Food Drug Anal. 22 (4) (2014) 407-412.

[70] J. Vial, K. Le Mapihan, A. Jardy, Chromatographia 57 (1) (2003) S303-S306.

[71] M. Ribani, C.H. Collins, C.B.G. Bottoli, J. Chromatogr. a 1156 (1-2) (2007) 201-205.

[72] A. Banel, M. Wasielewska, B. Zygmunt, Anal. Bioanal. Chem. 399 (9) (2011) 3299-3303.

[73] R. Sousa, V. Homem, J.L. Moreira, L.M. Madeira, A. Alves, Anal. Meth. 5 (11) (2013) 2736-2745.

[74] R. Perestrelo, A. Fernandes, F.F. Albuquerque, J.C. Marques, J.S. Câmara, Anal. Chim. Acta 563 (1-2) (2006) 154-164.

[75] V. Ferreira, R. López, J.F. Cacho, J. Sci. Food Agric. 80 (11) (2000) 1659-1667.

[76] E. Campo, V. Ferreira, A. Escudero, J.C. Marques, J. Cacho, Anal. Chim. Acta 563 (1-2) (2006) 180-187.

[77] K. Takahashi, N. Goto-Yamamoto, J. Chromatogr. a 1218 (43) (2011) 7850-7856.

[78] V. Pereira, M. Santos, J. Cacho, J.C. Marques, LWT - Food Sci. Technol, (Lebensmittel-Wissenschaft -Technol.) 75 (2017) 719-726.

[79] B. Gonçalves, V. Falco, J. Moutinho-Pereira, E. Bacelar, F. Peixoto, C. Correia, J. Agric. Food Chem. 57 (1) (2009) 265-273.

[80] E. Campo, V. Ferreira, A. Escudero, J.C. Marqués, J. Cacho, Anal. Chim. Acta 563 (1-2) (2006) 180-187. 